Published in final edited form as:

Nat Methods. 2018 February ; 15(2): 127-133. doi:10.1038/nmeth.4533.

\title{
Biotinylation by antibody recognition - A method for proximity labeling
}

Daniel Z Bar, National Human Genome Research Institute, National Institutes of Health, Bethesda, Maryland 20892, USA

\section{Kathleen Atkatsh,} National Human Genome Research Institute, National Institutes of Health, Bethesda, Maryland 20892, USA

\section{Urraca Tavarez,} National Human Genome Research Institute, National Institutes of Health, Bethesda, Maryland 20892, USA

\section{Michael R Erdos,} National Human Genome Research Institute, National Institutes of Health, Bethesda, Maryland 20892, USA

Yosef Gruenbaum, and Department of Genetics, Institute of Life Sciences, The Hebrew University of Jerusalem, Givat Ram, Jerusalem 9190401, Israel

\section{Francis S Collins}

National Human Genome Research Institute, National Institutes of Health, Bethesda, Maryland 20892, USA

\section{Abstract}

Identification of protein-protein interactions is a major goal of biological research. Despite technical advances over the last two decades, important but still largely unsolved challenges include the high-throughput detection of interactions directly from primary tissue and the identification of interactors of insoluble proteins that form higher-order structures. We have developed a novel, proximity-based labeling approach that uses antibodies to guide biotin deposition onto adjacent proteins in fixed cells and primary tissues. We showed our method to be specific and sensitive by labeling a mitochondrial matrix protein. Next, we used this method to profile the dynamic interactome of lamin $\mathrm{A} / \mathrm{C}$ in multiple cell and tissue types under various

\footnotetext{
Users may view, print, copy, and download text and data-mine the content in such documents, for the purposes of academic research, subject always to the full Conditions of use: http://www.nature.com/authors/editorial_policies/license.html\#terms

Corresponding authors: Correspondence should be addressed to Francis S. Collins (francis.collins@nih.gov).

Author Contributions

D.Z.B and F.S.C designed the experiments with input from Y.G., D.Z.B and K.A. performed the experiments, with assistance from M.R.E. U.T. performed the mouse work, D.Z.B analyzed the data. D.Z.B and F.S.C wrote the manuscript, with comments and edits by K.A and Y.G.

Competing financial interests

The National Institutes of Health has filed for a patent application covering some parts of the information contained in this article.
} 
treatment conditions. The ability to detect proximal proteins and putative interactors in intact tissues, and to quantify changes caused by different conditions or in the presence of disease mutations, can provide a new window into cell biology and disease pathogenesis.

\section{Introduction}

Protein-protein interactions (PPI) are critical to the function of all living cells. The protein interactome is dynamic: interactions may change with time, developmental stage, cell cycle progression, or tissue type. Characterizing PPI can provide important information about the locations and functions of a protein of interest. However, the effect of specific mutations on tissue-specific protein interactomes has rarely been studied in any real detail.

Distinct mutations within a single gene may result in a plethora of diseases. Over 400 different mutations of the lamin $\mathrm{A} / \mathrm{C}(L M N A)$ gene give rise to more than 14 distinct phenotypes that affect a variety of tissues - among them lipodystrophies, muscular dystrophies and premature aging syndromes. The mechanistic links between these singlegene mutations and their highly variable phenotypes remain to be elucidated. Tissue-specific interactors may offer an explanation, but characterization of the tissue-specific lamin interactome is challenging and has yet to be accomplished.

Analysis of the lamin interactome by standard Co-Immunoprecipitation (CoIP) is impractical because the insoluble high order structures created by the lamin filaments result in superfluous precipitation of unrelated proteins. Several attempts to identify lamin interactors and to characterize the protein composition of the NE have been made with various genetic and biochemical approaches (abbreviated BioID ${ }^{1}$, OneSTrEP-tag ${ }^{2}, \mathrm{Y}_{2} \mathrm{H}^{3}$, Lamin A/C tail ${ }^{4}$, Liver LB BioID ${ }^{5}$ and FACS + salt $^{6}$ ). While multiple known and novel interactors were identified by these methods, their applications were limited to single cell lines and showed only a modest overlap amongst different datasets (see below).

Previously published proximity-based labeling methods have generally required the prior insertion of a fusion gene in cell lines or in the germline, and thus cannot be used on primary human tissue samples. To overcome these limitations, we have developed an antibodyguided, proximity-based labeling method denoted Biotinylation by Antibody Recognition (BAR). We validated our method on a mitochondrial matrix protein and applied it to identify the interactors of lamin $\mathrm{A} / \mathrm{C}$ in immortalized cell lines, primary cell culture, and primary human muscle and adipose tissues. We further expanded the method to include differential proteomics and identified stress and mutation-induced changes to the composition of the nuclear envelope (NE).

\section{Results}

The BAR method is designed to identify proteins in the vicinity of an antigen (Fig. 1A). In a fixed and permeabilized tissue sample, a primary antibody is used to target a protein of interest. In the presence of hydrogen peroxide and phenol biotin, a secondary HRPconjugated antibody creates free radicals, resulting in biotinylation of proteins in close proximity to the target protein. As biotin is covalently attached, harsh conditions may then 
be used for reverse cross-linking and protein solubilization. Streptavidin-coated beads are used to precipitate the biotinylated proteins, which are then detected by tandem massspectrometry. When possible, Stable Isotope Labeling by Amino Acids in Cell Culture (SILAC) can be used to contrast signal coming from the target of interest with signal arising outside the region of interest.

\section{Estimation of specificity and sensitivity by mitochondrial proximity labeling}

We estimated the performance of our method on the mitochondrion, an organelle whose protein composition has been extensively characterized by multiple methods ${ }^{7-12}$. Of the 219 proteins having a heavy to light SILAC ratio above 2, $95 \%$ were known mitochondrial proteins (Fig. 1B, Supplementary Dataset: Mitochondria). Of these 219 proteins, $\sim 74 \%$ were known mitochondrial matrix proteins. Of all mitochondrial matrix proteins passing quantification criteria, $88 \%$ were included in the 219 list. Of note, we do not expect all known matrix proteins to be enriched in our sample, as for some of them, like GRHPR, most of the protein localizes outside the mitochondria. Of the 219 identified proteins, only $\sim 1.4 \%$ (3/219) were known non-mitochondrial proteins (i.e. potential false positives).

\section{Lamin A/C proximity labeling in HeLa cells}

We applied BAR to identify proteins in the vicinity of lamin A/C in HeLa cells. Biotin was successfully deposited at the NE, as evident from super-resolution microscopy (Fig. 1C). Biotinylated proteins were pulled down with streptavidin-coated beads and analysed by Western blot and mass-spectrometry of in-gel digested proteins (Supplementary Fig. 1A-C). In addition to identifying the targeted lamin $\mathrm{A} / \mathrm{C}$, we identified multiple known interactors of lamin A/C, including lamin B1, lamin B2 and lamina-associated polypeptide 2 (LAP2) (Supplementary Fig. 1D, Supplementary Dataset: HeLa In-Gel digestion).

\section{Ratiometric analysis}

To improve on these results, we sought to increase signal intensity. Reaction time determines the labeling radius and signal intensity. While limiting the labeling radius reduced the number of non-NE proteins identified, it also decreased the signal intensity both for rare and abundant NE proteins (Supplementary Fig. 2A). By contrast, increasing the reaction time results in signal arising from leakage to non-nuclear envelope proteins. To overcome this, we employed a ratiometric labeling ${ }^{11}$.

We employed two strategies: (i) As the majority of signal leakage, as well as signal originating from nucleoplasmic lamin, labels the nucleoplasm, we contrasted signal originating from the nuclear envelope with a signal of active chromatin (H3K27ac) using SILAC. (ii) An adjacent control can occasionally be found for some subcellular locations ${ }^{11}$, however this may not be the case for proteins with multiple subcellular locations or proteins found between distinct cellular structures. Thus, we also determined enrichment by comparing the streptavidin-bound fraction with the unbound fraction.

When contrasting with active chromatin (H3K27ac), known nuclear envelope interactors showed the highest SILAC ratios, with all three lamin proteins ranked in the top 6 (out of $\sim 1300$ proteins passing $3+$ peptides quantification criteria). Alternatively, comparing to the 
unbound fraction identified more (Fig. 1D) nuclear pore complex proteins, possibly due to inner-nuclear roles of some of these proteins. Compared to our "in-gel" dataset, ratiometric labeling resulted in a modest improvement in dataset quality and a significant improvement in protein signal and peptide count, thus enabling better quantification (Supplementary Dataset: HeLa LMNA-Unbound, HeLa LMNA-H3K27ac).

\section{Comparison with six published lamin interactomes}

We compared our BAR results to that obtained from six other methods ${ }^{1-6}$. The overlap between the six previously published datasets, while statistically significant, is small (Supplementary Dataset: Datasets aligned and Supplementary Fig. 2B). To gain a better estimate of coverage of true positive results, we defined high confidence interactors as proteins identified by three or more datasets. $81 \%$ (71/88) of these proteins were identified by our LMNA-H3K27ac dataset (Fig. 1E). Similar conclusions resulted from comparing the Gene Ontology enrichments of the various datasets (Fig. 1F).

\section{Labeling with different antibodies and other NE proteins}

To assess the role of antibody quality and binding site on the data generated, we performed SILAC experiments, labeling heavy and light cells with different lamin A/C antibodies. After normalization, the ratio for the majority of nuclear envelope proteins, and in particular proteins where quantification results were robust, did not significantly deviate from one (Supplementary Fig. 2C, Supplementary Dataset: LMNA antibody compare).

The reaction labeling radius does not enable the discrimination of proteomes of adjacent proteins. This can be an advantage, for example when studying how null mutations affect organelle composition by targeting an adjacent unchanged protein. B-type lamin filaments are in close proximity to lamin $\mathrm{A} / \mathrm{C}$ near the NE, but are not found in the nucleoplasm. Indeed, only modest differences in protein abundance were observed (Supplementary Fig. 2D, Supplementary Dataset: NE protein compare). Overall these results suggest that antibody localization, and not protein identity or binding epitope, is the primary determinant of the protein identified.

\section{Lamin $A / C$ proximity labeling in primary human tissue}

An underlying assumption of cell culture usage is that it mimics relevant processes that occur throughout the entire organism. While this assumption is often valid, in many cases it is not. To overcome such limitations, we attempted to identify lamin A/C interactors in primary human tissue relevant to laminopathies. We successfully labeled the NE in postmortem tissue samples. Nuclear envelope morphology in these samples deviated considerably from the classical view of a smooth round sphere around the nucleus. For example, muscle myofibrils created grooves in the nucleus, which correlate not only with the distribution of lamin A/C, but also of DNA (Fig. 2A, Supplementary Fig. 3A). Some nuclei in adipose had a doughnut shape (Fig. 2B, Supplementary Fig. 3B), a phenotype that was previously reported in cell culture but not in primary human tissues ${ }^{13}$. While different tissues had multiple lamin $\mathrm{A} / \mathrm{C}$ interactors in common, some proteins were either found exclusively or with greater abundance in a particular tissue. For example, Muscular LMNAinteracting protein (MLIP) was found in all muscle samples but not in HeLa or adipose 
samples (Supplementary Dataset). Proteins identified by two or more unique peptides in one experiment had a $\sim 90 \%$ chance of being detected (at any level) in a replicate experiment (Fig. 2C). However, signal correlation between replications, while highly statistically significant, was insufficient to quantify small differences without multiple repetitions (Fig. 2D). Overall, skeletal muscle tissue showed higher similarity to smooth muscle tissue than to adipose tissue (Fig. 2E). These results provide further evidence that NE composition varies between tissues ${ }^{14}$.

Muscle tissues showed NE enrichment for several proteins mutated in muscular dystrophies and cardiomyopathies, including multiple members of the dystrophin-glycoprotein complex (see muscle samples in Supplementary Dataset). While members of this complex were not previously reported to be associated with lamin $\mathrm{A} / \mathrm{C}$, fluorescent protein fusion and antibody staining in cell culture ${ }^{15}$ suggests that this complex is enriched at close proximity to the NE, presumably in the perinuclear ER (Fig. 2F and Supplementary Fig. 3C). Similar findings are seen in some muscle and non-muscle sarcoglycan (SGCA) stained sections in the protein atlas project ${ }^{16}$. In support of our hypothesis, lamin $\mathrm{A} / \mathrm{C}$ was also moderately enriched in primary skeletal muscle when BAR was applied with an antibody against SGCA (Supplementary Dataset: Human Skeletal Muscle SGCA).

\section{Differential proteomics}

Qualitative and quantitative changes may be observed when comparing the protein interactome under different conditions, such as the presence or absence of a genetic variation, application of a treatment protocol, or variation of a tissue type. Identifying such changes is important for understanding the function of the target protein in that context. While a protein of interest may be in close proximity to multiple other proteins, only a small fraction of those proteins may be relevant to a specific function or process, and this fraction is more likely to change in that context. As a proof of concept for differential proteomics, we compared the NE composition of naive HeLa cells with cells subjected to a 2-hour $43^{\circ} \mathrm{C}$ heat shock. As expected, in control (naive) cells, the heavy to light ratio was very close to one, particularly when multiple peptides were used to calculate the ratio (Supplementary Fig. 4A-B, Supplementary Dataset: No Treatment SILAC). By contrast, when comparing a control with a heat shocked sample, multiple proteins displayed deviant heavy to light ratios (Fig. 3A - blue bars). These changes were mirrored in a reciprocal experiment (Fig. 3A - red bars). Proteins identified include expected targets, such as multiple members of the heat shock protein family. As these proteins become abundant during heat shock conditions, the NE bound fraction is also expected to increase. By contrast, we also identified elevated levels of non heat-shock proteins. For example, Ku70 and Ku80 (also known as XRCC6 and XRCC5, respectively) are regulators of DNA-PK catalytic subunit (DNA-PKcs, also known as PRKDC) and members of the non-homologous end joining pathway. We found Ku70 and Ku80 to be $~ 2.5$-fold more abundant near the NE following heat shock (Fig 3A and Supplementary Dataset: Heat Shock Vs. Control). In contrast, no enrichment was seen for DNA-PKcs. We validated the conclusion that Ku70 and Ku80 localize near lamin A/C using Förster resonance energy transfer (FRET) (Supplementary Fig. 5) and also observed an increase in Ku70 and Ku80 nuclear peripheral localization using immunofluorescence following heat shock (Fig. 3B, Supplementary Fig. 6A). Ku70 was previously identified ${ }^{1,2}$ 
as a lamin $\mathrm{A} / \mathrm{C}$ binding protein and heat shock inactivates Ku 80 and drives its aggregation ${ }^{17}$. HSPA8, known to bind Ku70 and suppress its nuclear import, also showed a similar NE increase (Fig 3A, Supplementary Fig. 6A and Supplementary Dataset: Heat Shock Vs. Control). These changes reflect changes in molecular localization, and not total protein abundance (Supplementary Fig. 6B and Beck et al. ${ }^{17}$ ).

\section{Progerin proximity labeling in transfected HeLa cells}

Roughly $90 \%$ of cases of the premature aging disease Hutchinson Gilford progeria syndrome (HGPS) are caused by a de-novo synonymous mutation of the lamin A/C gene ${ }^{18}$. This mutation activates a cryptic splice site, resulting in a protein lacking 50 amino acids near the C-terminus, termed progerin. We applied BAR to HeLa cells transfected with GFPLMNA or GFP-progerin and used a GFP antibody to direct biotin labeling. By observing the cells 24 hours after transfection (Fig. 3C, Supplementary Dataset: GFP Progerin), we were able to detect changes to the composition of the NE resulting directly from the acute expression of progerin. As expected, the heavy to light ratio for most lamin $\mathrm{A} / \mathrm{C}$ peptides was close to one. Only three peptides exhibited a significant deviation from this ratio, all of which overlapped with the exon 11-encoded 50 amino acid stretch found only in lamin A/C and not in progerin, thus demonstrating our ability to distinguish between isoforms even in a wild-type lamin A/C endogenous background (Fig. 3D). Among the proteins preferentially binding progerin was DNA-PKcs (Fig. 3E), previously reported to bind progerin ${ }^{19}$.

\section{Nuclear envelope proximity labeling in primary HGPS fibroblasts}

To detect changes in NE composition resulting from progerin expression in primary tissue culture, we compared the NE composition of control fibroblasts with fibroblasts donated by a HGPS patient (Fig. 3F, Supplementary Dataset: HGPS NE Comparison) We detected changes in the abundance of several known NE proteins, including decrease of lamin B2 and RANBP2 and an increase in nesprin-1 and SUN2 (Fig. 3G and Supplementary Dataset: HGPS NE Comparison). Additionally, in HGPS fibroblasts we saw a NE decrease in Polymerase I and transcript release factor (PTRF, also known as CAVIN1) and its interactor caveolin-1 (CAV1). Mutations in PTRF and Caveolin family member proteins are associated with lipodystrophy and muscular dystrophy ${ }^{20}$. Only a small fraction of CAV1 localizes near the nuclear membrane and may be available for NE interactions (Supplementary Fig. 7A). As CAV1 was highly enriched in our muscle NE datasets, we looked at this more biologically relevant tissue. Indeed, a significant fraction of CAV1 is found at the nuclear membrane (Fig 3H, Supplementary Fig. 7B) in muscle tissues.

\section{Discussion}

We have developed a novel and generalizable method for the identification of proteins in close proximity to an antigen, validated the sensitivity and specificity of the method by applying it to mitochondria, and successfully applied it to characterize the NE composition in multiple cell lines and tissues. 


\section{Advantages and Limitations of the Proximity-based Labeling Method}

By replacing enzyme fusion with antibodies, our method offers several advantages. BAR does not need a separate cell line or animal model to be generated for every protein of interest. Our use of antibodies prevents any protein fusion related artifacts. Nonetheless, our method has several drawbacks. These include the requirement for a monospecific antibody that works well in tissue sections and sensitivity to fixation artifacts. Finally, our label-free quantification does not allow the detection of small changes in differential proteomics. Future studies and the online protocol we have deposited now suggest the use of isobaric mass tags (TMT).

\section{Tissue specificity}

Previous studies characterizing the lamin interactome analyzed single cell lines. To gain insight into how specific mutations in lamin $\mathrm{A} / \mathrm{C}$ cause multiple distinct clinical conditions, we analyzed the lamin interactome of multiple cell lines and primary human tissues. Certain proteins known to be clinically relevant, which were not identified by previous studies nor by BAR as lamin A/C interactors in HeLa cells, were identified by BAR in disease-relevant primary tissues. For example, primary muscle tissue shows NE enrichment for members of the dystrophin-glycoprotein complex. Mutations in this complex can result in various muscle dystrophies that resemble the muscle dystrophies caused by lamin A/C mutations. We show that multiple members of the dystrophin-glycoprotein complex are found in close proximity to the NE in skeletal muscle (Fig. 2F, Supplementary Fig. 3C, Supplementary Dataset: Human Skeletal Muscle). Interestingly, mutations to the dystrophin protein can cause NE abnormalities that progress with cell passage ${ }^{21}$. The dystrophin-glycoprotein complex has a structural role in linking the cytoskeleton to the extracellular matrix. In view of its enrichment at the nuclear periphery (Fig. 2F, ref. ${ }^{15}$ ) and the nuclear deformations caused by DMD mutations ${ }^{21}$, we speculate that this complex may have a structural role in regulating NE morphology and nuclear position in muscle tissue. As multiple members of the dystrophin-glycoprotein complex are transmembrane proteins, it is even possible that the complex penetrates into the perinuclear space, where it may facilitate interactions with proteins of the inner nuclear membrane. In support of this hypothesis, mutations to dystrophin or $\delta$-sarcoglycan alter the distribution of NE proteins lamin A/C, Emerin and and Nesprin-2 $2^{21,22}$.

\section{Differential proteomics}

Comparing the interactome of a target protein under various conditions can help identify interactions that are important for a specific function. In HeLa cells expressing progerin or in HGPS fibroblasts, we were able to replicate many known changes to the NE composition, as well as identify novel disease-relevant proteins. These include RanBP2 and Linker of Nucleoskeleton and Cytoskeleton (LINC) complex members nesprin-1 and SUN2, all of which have important roles in laminopathies ${ }^{23-27}$, as well as PTRF, not previously known to associated with progerin. Interestingly, lamin A is known to regulate $P T R F$ transcription $^{28}$ and PTRF and CAV1 inhibit NRF2, promoting stress-induced premature senescence ${ }^{29}$. We note that while PTRF and CAV1 peptides are hardly seen in HeLa cells, these proteins gave a robust signal in fibroblasts, as well as primary muscle tissue. Moreover, clear nuclear 
membrane localization of CAV1 was seen only in muscle samples (Fig. 3H), emphasizing the need to explore changes to the NE composition in a relevant tissue.

In future studies, this method can be leveraged to characterize the interactome of various proteins directly from clinical samples of patients and controls, thus accounting for the effect of both genetic and non-genetic factors, like a patient's life history, on the interactome. Furthermore, BAR can be used to identify interactors of modified proteins or other biomolecules simply by using the appropriate antibody to target the molecule of interest.

\section{Material and Methods}

A list of antibodies, plasmids and cell lines used is presented in Supplementary Table 12-34.

\section{Tissue Culture}

Cells were grown without antibiotics as described below. All cell lines were tested for mycoplasma (MycoFluor Mycoplasma detection kit, Invitrogen). Fibroblasts cell identity was further validated by analyzing deep-sequencing data generated for other projects.

\section{Biotinylation by Antibody Recognition}

A complete protocol is available online. Briefly, samples were fixed in $4 \%$ formaldehyde (Thermo Fisher Scientific) for 10-30 minutes at room temperature (RT) and washed with PBST (PBS with $0.1 \%$ tween 20). Samples were incubated with $0.5 \%$ hydrogen peroxide for 10 minutes to deactivate any endogenous peroxidase activity. Samples were then permeabilized in PBS with $0.5 \%$ triton X-100 for 7 minutes and blocked for 2 hours in $1 \%$ bovine serum albumin (BSA) in PBST. Samples were incubated with primary antibody overnight, washed with PBST, and incubated with an appropriate secondary antibody conjugated to horseradish peroxidase (HRP) for 3 hours. After extensive washes, samples were incubated with biotin-tyramide (Perkin Elmer) for 10 minutes and a dilution buffer containing hydrogen peroxide was added to a total volume of $150 \mu \mathrm{l}$. The reaction was stopped after 1-7 minutes (see Supplementary Dataset: Index) by adding $850 \mu \mathrm{l}$ of $500 \mathrm{mM}$ sodium ascorbate (Sigma-Aldrich). After two washes with PBST, sample subsets were incubated with FITC-avidin and analyzed under a microscope to validate the expected staining pattern of a given antibody. The remainder of the samples were heated to $99^{\circ} \mathrm{C}$ for an hour with $1.5 \%$ SDS and $1 \%$ sodium deoxycholate. Sample volume was adjusted to $1 \mathrm{ml}$ with PBST and biotinylated proteins were extracted with streptavidin beads (Thermo Fisher Scientific) according to the manufacturer's protocol. For some samples, the presence of a specific protein bound to the beads was validated by Western blot using $\sim 10 \%$ of the beads. Samples were prepared for LC-MS/MS by incubation for 30 minutes at $37^{\circ} \mathrm{C}$ in $10 \mathrm{mM}$ DTT (Thermo Fisher Scientific) followed by 20 minutes in $50 \mathrm{mM}$ Iodoacetamide (Thermo Fisher Scientific) at room temperature protected from light. Finally, samples were digested overnight in $37^{\circ} \mathrm{C}$ with $2 \mu \mathrm{g}$ trypsin (Promega), followed by a second 2 hour digestion.

\section{Enrichment analysis}

For non-SILAC samples, the enrichment factor (E) is set to be $\min \left(\mathrm{E}_{\mathrm{No}} \mathrm{Ab}, \mathrm{E}_{\text {Unbound }}\right)$, where $\mathrm{E}_{\mathrm{No}} \mathrm{Ab}$ is the area ratio of the bound to no antibody control (non-specific binding to beads), 
and $\mathrm{E}_{\mathrm{Unbound}}$ is the area ratio of the bound to unbound fractions, normalized to the total area ratios. For proteins found only in the bound sample, E was arbitrarily set to 1000. If the area could be calculated for the bound but not the control sample, E was arbitrarily set to 100 . Surprisingly, E was often lower for the target protein than for other known interactors. We speculate this is due to multiple biotinylations, that decreases peptide identification by massspectrometry. Known contaminants are shown in the raw data but removed from final lists and analysis.

In SILAC samples comparing different mutations or cell lines, protein quantities were normalized, setting the heavy/light average or lamin $\mathrm{A} / \mathrm{C}$ ratio to 1 .

\section{Transgene expression}

HeLa cells grown in T-75 flasks were transfected using $7 \mu \mathrm{g}$ per flask of plasmid DNA with lipofectamine 2000 (Thermo Fisher Scientific) in accordance with the manufacturer's instructions.

\section{Stable Isotope Labeling by Amino acids in Cell culture}

Cells were grown in SILAC MEM medium (Thermo Fisher Scientific) lacking lysine and arginine, supplemented with dialysed 10\% FBS (Sigma-Aldrich), $200 \mathrm{mg} / \mathrm{l}$ light L-Proline (Sigma-Aldrich) and either heavy L-Lysine $\left({ }^{13} \mathrm{C}_{6}{ }^{15} \mathrm{~N}_{2} ; 146 \mathrm{mg} / \mathrm{l}\right)$ and L-Arginine $\left.{ }^{13} \mathrm{C}_{6}{ }^{15} \mathrm{~N}_{4} ; 84 \mathrm{mg} / \mathrm{l}\right)($ Cambridge Isotope Laboratories) or their light equivalents (SigmaAldrich). Cells were passaged with non-enzymatic Gibco Cell Dissociation Buffer (Thermo Fisher Scientific). $>95 \%$ heavy amino acid incorporation rate was validated by massspectrometry.

\section{LC-MS/MS analysis}

Protein identification by LC-MS/MS analysis of peptides was performed using an Eksigent nanoLC-Ultra 1D plus system (Dublin, CA) coupled to an LTQ Orbitrap Elite or Fusion Lumos mass spectrometer (Thermo Fisher Scientific, San Jose, CA) using CID fragmentation. Peptides were first loaded onto a Zorbax 300SB-C18 trap column (Agilent, Palo Alto, CA) at a flow rate of $6 \mu \mathrm{l} / \mathrm{min}$ for $6 \mathrm{~min}$, and then separated on a reversed-phase PicoFrit analytical column (New Objective, Woburn, MA) using a 120-min linear gradient of $5-35 \%$ acetonitrile in $0.1 \%$ formic acid at a flow rate of $250 \mathrm{nl} / \mathrm{min}$. LTQ-Orbitrap Elite settings were as follows: spray voltage $1.5 \mathrm{kV}$; full MS mass range $\mathrm{m} / z 300$ to 2,000. The LTQ-Orbitrap Elite was operated in a data-dependent mode; i.e., one MS1 high resolution $(60,000)$ scan for precursor ions is followed by six data-dependent MS2 scans for precursor ions above a threshold ion count of 500 with collision energy of $35 \%$.

\section{Database search criteria}

Raw files generated by the LTQ Orbitrap Elite or Orbitrap Fusion Lumos were analyzed using Proteome Discoverer v1.4 software (Thermo Fisher Scientific) using Mascot (Matrix Science, London, UK; version 2.5.1) or SEQUEST search engines. The search criteria were set to: database, Swiss Prot (Swiss Institute of Bioinformatics); taxonomy, Human or Mouse; enzyme, trypsin; miscleavages, 2; variable modifications, Oxidation (M), Deamidation (NQ), isotope labeling of lysine $(\mathrm{K}+8.014 \mathrm{Da})$ and arginine $(\mathrm{R}+10.008 \mathrm{Da})$; 
fixed modifications, Carbamidomethyl (C); MS peptide tolerance $20 \mathrm{ppm}$; MS/MS tolerance as $0.8 \mathrm{Da}$. For the in-gel digestion dataset, identifications were accepted based on one or more unique peptides with a false discovery rate (FDR) of $99 \%$ or higher. All other datasets accepted based on two or more unique peptides with a false discovery rate (FDR) of 99\% or higher.

\section{Statistics and data analysis}

Datasets were imported into a MySQL database. Comparisons involving external datasets was done by converting gene names into the HGNC (http://www.genenames.org/) standard or Uniprot accession (mitochondria data only). $P$-values for single proteins were calculated using one-sided $t$-test on peptide SILAC ratios. Investigators were not blinded to group allocations. Additional information can be found online at the Life Science Reporting Summary.

\section{Human tissue samples}

Cadaveric adipose and muscle tissues used in this study were obtained from the National Disease Research Interchange (NDRI) biorepository, exempted by the NIH Office of Human Subjects Research.

\section{Mouse tissue preparation}

All animal experiments were approved by NHGRI animal care and use committee. Experiments were performed according to institutional guidelines in accordance with The Guide for the Care and Use of Laboratory Animals and the AVMA guidelines for the Euthanasia of Animals.

C57BL/6 mice were housed in a AAALAC Accredited SPF Animal facility.

Perfusion was performed on 4 month old males by achieving a surgical plane of anesthesia characterized by loss of consciousness, loss of reflex muscle response and loss of response to noxious stimuli in accordance with the AVMA guidelines for the Euthanasia of Animals using Tribromoethanol (Avertin 1.25\%, $0.04 \mathrm{ml} / \mathrm{g} \mathrm{BW}$ IP). For mass-spectrometry experiments, perfusion of PBS ( $\mathrm{pH}$ 7.2) followed by $2 \%$ Paraformaldehyde in PBS was used for continuous fixation for 60 minutes. Extracted tissues were flash frozen in liquid nitrogen.

\section{Heat shock}

T75 Flasks of SILAC labelled HeLa cells were transferred to an incubator preheated to $43^{\circ} \mathrm{C}$ for two hours and immediately processed.

\section{Microscopy}

Panel 1C was acquired with a ZEISS LSM 880 with Airyscan system equipped with a PlanApochromat 63×/1.40 Oil DIC M27 objective and using 488 and $561 \mathrm{~nm}$ wavelength lasers. Panels 2A,B S3A, S6A and S7B were acquired with a DeltaVision PersonalDV (Applied Precision) with either a Plan Apo 60x/1.42 NA or a UPlanSApo 100x/1.4 NA oil lenses. Panels 2F, 3B,H, S3B,C, S5 and S7A were acquired on a Leica TCS SP5 confocal microscope equipped with a HCX PL APO CS 63.0x/1.40 NA oil lens. FRET experiments 
were performed on HeLa cells using the Leica FRET AB wizard. Ku70(AF488)/Ku80(488

DyLight)/DNA-PKcs(488 DyLight) served as donors and LMNA (AF594 for Ku80 and

DNA-PKcs FRET; AF555 for Ku70 FRET) as acceptor. Panels 3C,F were acquired with a

OMX Structured Illumination Super-resolution Scope equipped with a PlanApo 60x/1.40

Oil DIC objective and using 488 and $568 \mathrm{~nm}$ wavelength lasers.

\section{Data availability}

The data that support the findings of this study are available from the corresponding author upon request. A detailed protocol is available at the Protocol Exchange. The mass spectrometry proteomics data have been deposited to the ProteomeXchange Consortium via the PRIDE $^{35}$ partner repository with the dataset identifier PXD004736.

\section{Supplementary Material}

Refer to Web version on PubMed Central for supplementary material.

\section{Acknowledgments}

We thank Sajni Patel and Marjan Gucek of the NHLBI Proteomics Core for operation of the mass-spectrometer and technical assistance in sample preparation, Stephen M. Wincovitch for microscopy assistance and Julia Fekecs for preparing figure 1A. We thank Lori Bonnycastle and Amanda Dubose for critical reading of the manuscript, useful suggestions and comments.

\section{References}

1. Roux KJ, Kim DI, Raida M, Burke B. A promiscuous biotin ligase fusion protein identifies proximal and interacting proteins in mammalian cells. J Cell Biol. 2012; 196:801-810. DOI: 10.1083/jcb. 201112098 [PubMed: 22412018]

2. Kubben N, et al. Identification of differential protein interactors of lamin A and progerin. Nucleus. 2010; 1:513-525. DOI: 10.4161/nucl.1.6.13512 [PubMed: 21327095]

3. Dittmer TA, et al. Systematic identification of pathological lamin A interactors. Mol Biol Cell. 2014; 25:1493-1510. DOI: 10.1091/mbc.E14-02-0733 [PubMed: 24623722]

4. Depreux FF, et al. Disruption of the lamin A and matrin-3 interaction by myopathic LMNA mutations. Hum Mol Genet. 2015; 24:4284-4295. DOI: 10.1093/hmg/ddv160 [PubMed: 25948554]

5. Fu Y, et al. MacroH2A1 associates with nuclear lamina and maintains chromatin architecture in mouse liver cells. Sci Rep. 2015; 5:17186. [PubMed: 26603343]

6 . Engelke R, et al. The quantitative nuclear matrix proteome as a biochemical snapshot of nuclear organization. J Proteome Res. 2014; 13:3940-3956. DOI: 10.1021/pr500218f [PubMed: 25090448]

7. Calvo SE, Clauser KR, Mootha VK. MitoCarta2.0: an updated inventory of mammalian mitochondrial proteins. Nucleic Acids Res. 2016; 44:D1251-1257. DOI: 10.1093/nar/gkv1003 [PubMed: 26450961]

8. Pagliarini DJ, et al. A mitochondrial protein compendium elucidates complex I disease biology. Cell. 2008; 134:112-123. DOI: 10.1016/j.cell.2008.06.016 [PubMed: 18614015]

9. Smith AC, Robinson AJ. MitoMiner v3.1, an update on the mitochondrial proteomics database. Nucleic Acids Res. 2016; 44:D1258-1261. DOI: 10.1093/nar/gkv1001 [PubMed: 26432830]

10. Rhee HW, et al. Proteomic mapping of mitochondria in living cells via spatially restricted enzymatic tagging. Science. 2013; 339:1328-1331. DOI: 10.1126/science.1230593 [PubMed: 23371551]

11. Hung V, et al. Proteomic mapping of the human mitochondrial intermembrane space in live cells via ratiometric APEX tagging. Mol Cell. 2014; 55:332-341. DOI: 10.1016/j.molcel.2014.06.003 [PubMed: 25002142] 
12. Forner F, Foster LJ, Campanaro S, Valle G, Mann M. Quantitative proteomic comparison of rat mitochondria from muscle, heart, and liver. Molecular \& cellular proteomics : MCP. 2006; 5:608619. DOI: 10.1074/mcp.M500298-MCP200 [PubMed: 16415296]

13. Verstraeten VL, et al. Protein farnesylation inhibitors cause donut-shaped cell nuclei attributable to a centrosome separation defect. Proc Natl Acad Sci U S A. 2011; 108:4997-5002. DOI: 10.1073/ pnas.1019532108 [PubMed: 21383178]

14. Worman HJ, Schirmer EC. Nuclear membrane diversity: underlying tissue-specific pathologies in disease? Curr Opin Cell Biol. 2015; 34:101-112. DOI: 10.1016/j.ceb.2015.06.003 [PubMed: 26115475]

15. Draviam RA, Shand SH, Watkins SC. The beta-delta-core of sarcoglycan is essential for deposition at the plasma membrane. Muscle Nerve. 2006; 34:691-701. DOI: 10.1002/mus.20640 [PubMed: 17036316]

16. Uhlen M, et al. Towards a knowledge-based Human Protein Atlas. Nature biotechnology. 2010; 28:1248-1250. DOI: $10.1038 /$ nbt1210-1248

17. Beck BD, Dynlacht JR. Heat-induced aggregation of XRCC5 (Ku80) in nontolerant and thermotolerant cells. Radiat Res. 2001; 156:767-774. [PubMed: 11741501]

18. Eriksson M, et al. Recurrent de novo point mutations in lamin A cause Hutchinson-Gilford progeria syndrome. Nature. 2003; 423:293-298. DOI: 10.1038/nature01629 [PubMed: 12714972]

19. Liu GH, et al. Recapitulation of premature ageing with iPSCs from Hutchinson-Gilford progeria syndrome. Nature. 2011; 472:221-225. DOI: 10.1038/nature09879 [PubMed: 21346760]

20. de Haan W. Lipodystrophy and muscular dystrophy caused by PTRF mutations. Clin Genet. 2010; 77:436-437. DOI: 10.1111/j.1399-0004.2009.01365_3.x [PubMed: 20447152]

21. Taranum S, et al. LINC complex alterations in DMD and EDMD/CMT fibroblasts. Eur J Cell Biol. 2012; 91:614-628. DOI: 10.1016/j.ejcb.2012.03.003 [PubMed: 22555292]

22. Heydemann A, Demonbreun A, Hadhazy M, Earley JU, McNally EM. Nuclear sequestration of delta-sarcoglycan disrupts the nuclear localization of lamin A/C and emerin in cardiomyocytes. Hum Mol Genet. 2007; 16:355-363. DOI: 10.1093/hmg/ddl453 [PubMed: 17164264]

23. Kandert S, et al. Nesprin-2 giant safeguards nuclear envelope architecture in LMNA S143F progeria cells. Hum Mol Genet. 2007; 16:2944-2959. DOI: 10.1093/hmg/ddm255 [PubMed: 17881656]

24. Lei K, et al. Inner nuclear envelope proteins SUN1 and SUN2 play a prominent role in the DNA damage response. Curr Biol. 2012; 22:1609-1615. DOI: 10.1016/j.cub.2012.06.043 [PubMed: 22863315]

25. Starr DA. Laminopathies: too much SUN is a bad thing. Curr Biol. 2012; 22:R678-680. DOI: 10.1016/j.cub.2012.06.070 [PubMed: 22974992]

26. Datta S, Snow CJ, Paschal BM. A pathway linking oxidative stress and the Ran GTPase system in progeria. Mol Biol Cell. 2014; 25:1202-1215. DOI: 10.1091/mbc.E13-07-0430 [PubMed: 24523287]

27. Nikolova-Krstevski V, et al. Nesprin-1 and actin contribute to nuclear and cytoskeletal defects in lamin A/C-deficient cardiomyopathy. J Mol Cell Cardiol. 2011; 50:479-486. DOI: 10.1016/ j.yjmcc.2010.12.001 [PubMed: 21156181]

28. Swift J, et al. Nuclear lamin-A scales with tissue stiffness and enhances matrix-directed differentiation. Science. 2013; 341:1240104. [PubMed: 23990565]

29. Volonte D, et al. Inhibition of nuclear factor-erythroid 2-related factor (Nrf2) by caveolin-1 promotes stress-induced premature senescence. Mol Biol Cell. 2013; 24:1852-1862. DOI: 10.1091/mbc.E12-09-0666 [PubMed: 23637463]

30. Strambio-De-Castillia C, Niepel M, Rout MP. The nuclear pore complex: bridging nuclear transport and gene regulation. Nat Rev Mol Cell Biol. 2010; 11:490-501. DOI: 10.1038/nrm2928 [PubMed: 20571586]

31. Mi H, Muruganujan A, Casagrande JT, Thomas PD. Large-scale gene function analysis with the PANTHER classification system. Nat Protoc. 2013; 8:1551-1566. DOI: 10.1038/nprot.2013.092 [PubMed: 23868073] 
32. Zwerger M, et al. Altering lamina assembly reveals lamina-dependent and -independent functions for A-type lamins. J Cell Sci. 2015; 128:3607-3620. DOI: 10.1242/jcs.171843 [PubMed: 26275827]

33. Britton S, Coates J, Jackson SP. A new method for high-resolution imaging of Ku foci to decipher mechanisms of DNA double-strand break repair. J Cell Biol. 2013; 202:579-595. DOI: 10.1083/ jcb.201303073 [PubMed: 23897892]

34. Cao K, Capell BC, Erdos MR, Djabali K, Collins FS. A lamin A protein isoform overexpressed in Hutchinson-Gilford progeria syndrome interferes with mitosis in progeria and normal cells. Proc Natl Acad Sci U S A. 2007; 104:4949-4954. DOI: 10.1073/pnas.0611640104 [PubMed: 17360355]

35. Vizcaino JA, et al. 2016 update of the PRIDE database and its related tools. Nucleic acids research. 2016; 44:D447-456. DOI: 10.1093/nar/gkv1145 [PubMed: 26527722] 

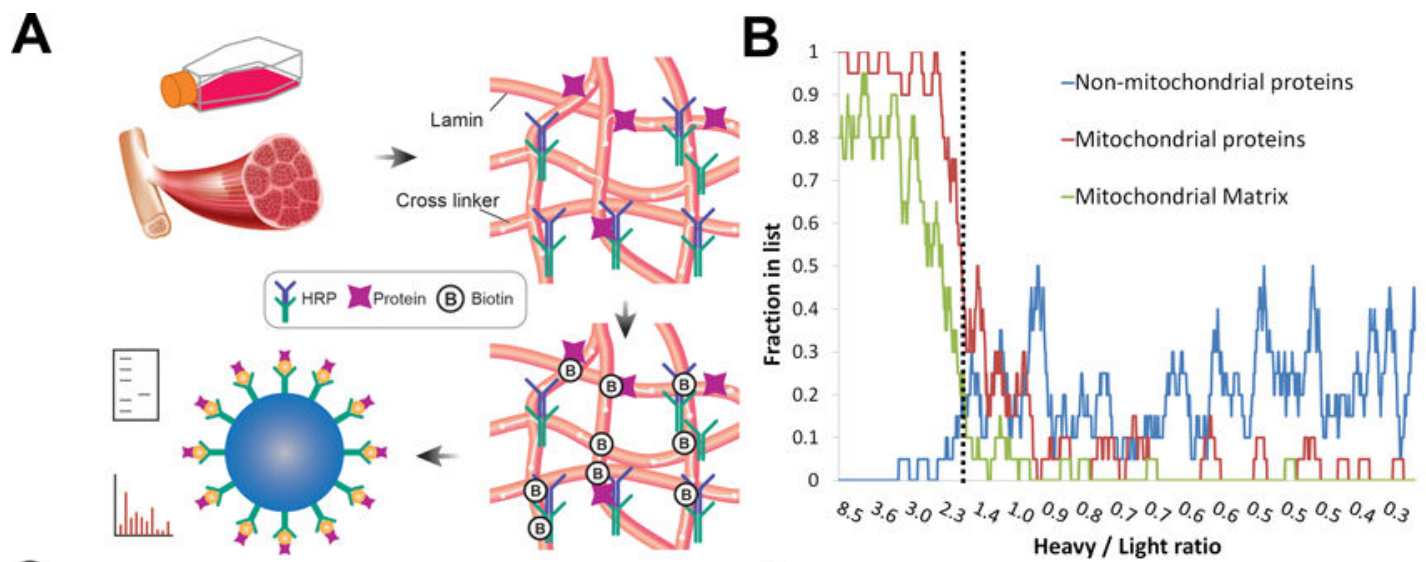

C
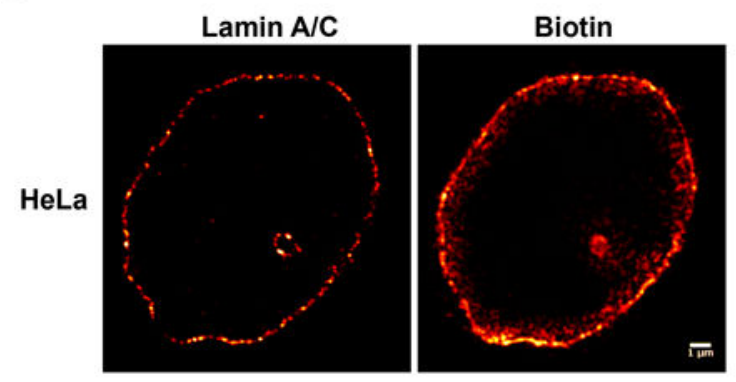

D

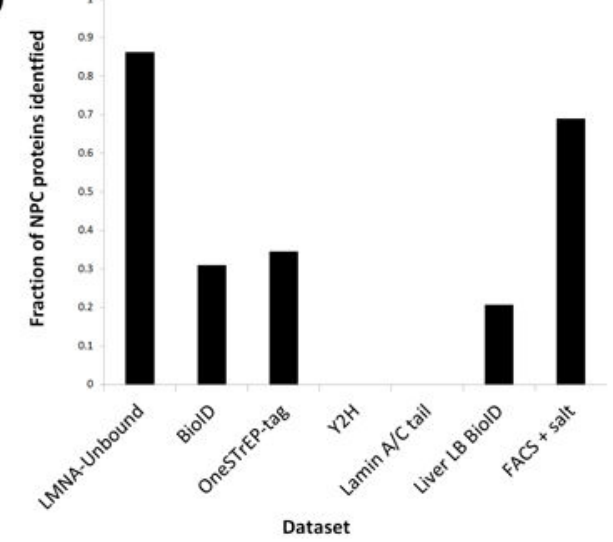

$\mathbf{E}$

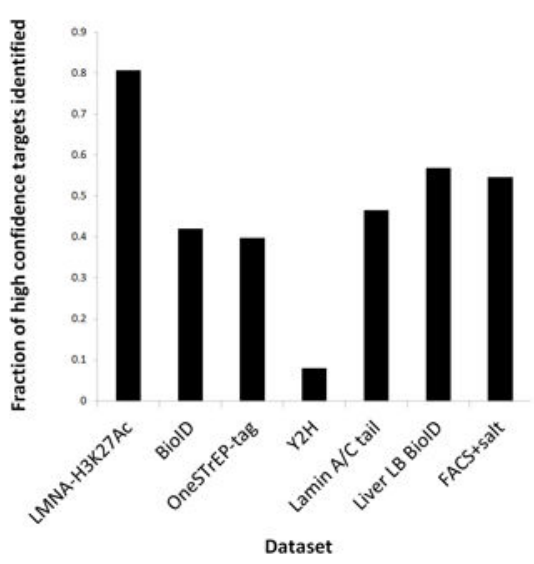

$\mathbf{F}$

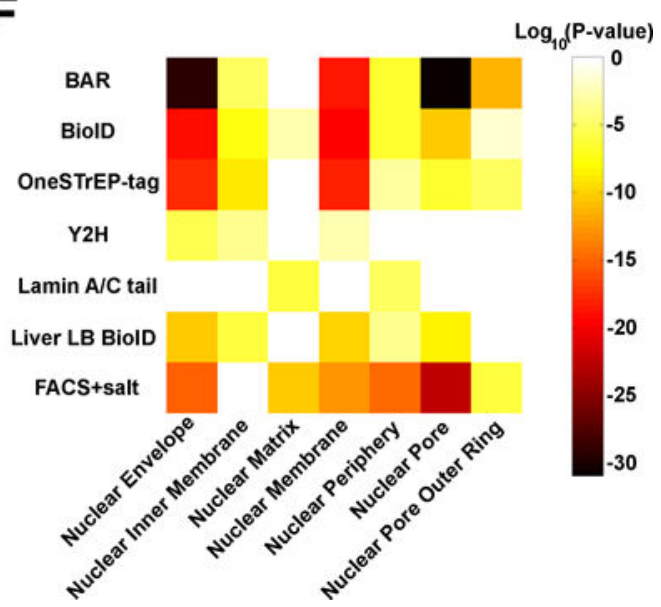

Figure 1. Biotinylation by antibody recognition

A. Schematic representation of the method, applied in this case to lamin A/C.

B. Proteins were ordered by descending SILAC ratio ( $\mathrm{x}$ - axis). Using a 20-proteins sliding window, fractions shared with each of the three datasets ${ }^{8-10}$ are plotted.

C. Super-resolution microscopy showing biotin deposition in the vicinity of the NE, confirming the tight spatial resolution of the protein labeling method. Scale bar - $1 \mu \mathrm{m}$.

D. The fraction of 29 known nuclear core complex proteins ${ }^{30}$ identified in each dataset is shown. 
E. The fraction of high confidence targets identified in each dataset is shown.

F. Heat map showing P-values for 7 Gene Ontology cellular component categories across our LMNA-Unbound dataset, as well as the 6 other published datasets. P-values were calculated using PANTHER overrepresentation test ${ }^{31}$. 
A

Smooth Muscle

Lamin A/C

Biotin

DNA

Merged

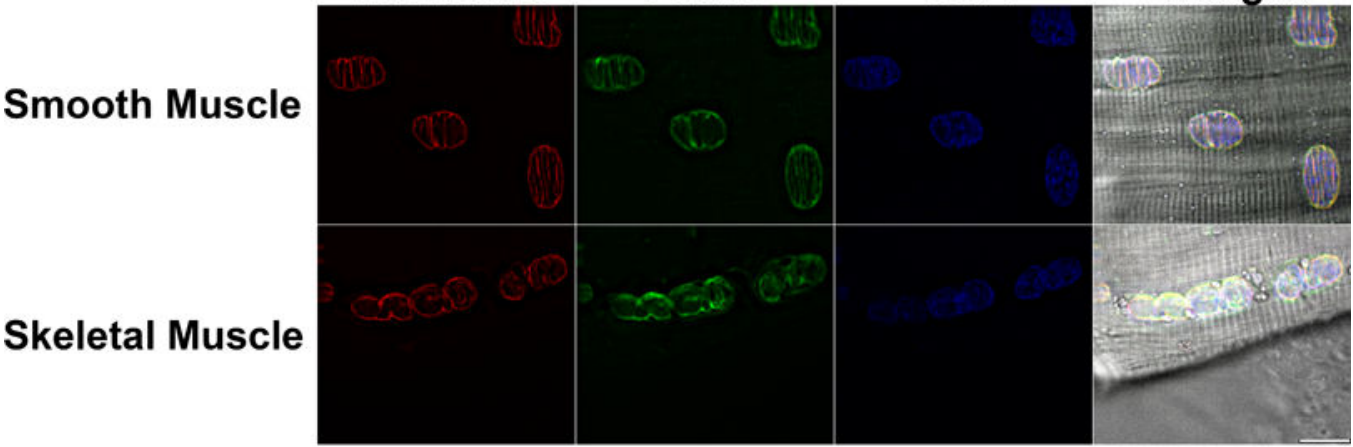

B

C

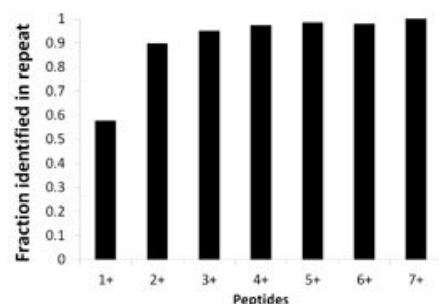

D

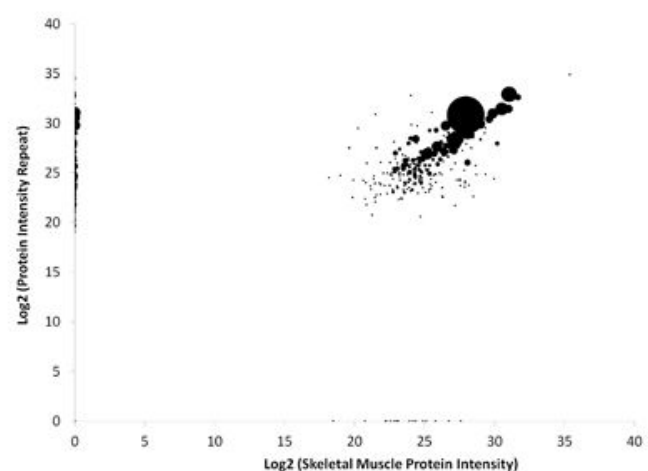

E

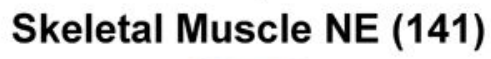

$\mathbf{F}$

SGCA

Skeletal Muscle

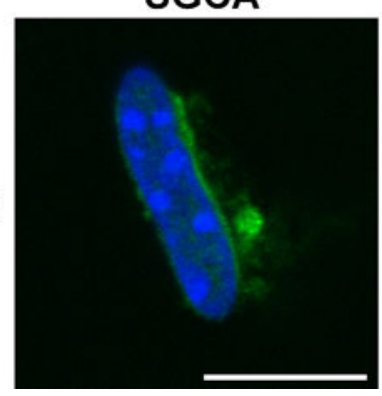

Figure 2. Identification of $\mathrm{NE}$ proteins in primary human tissue

A. Imaging of primary human skeletal and smooth muscle tissue.

B. Doughnut shaped nuclei from primary human adipose tissue.

C. Fraction of proteins with enrichment score $>1$ identified in a repeated human skeletal muscle BAR experiment, as a function of unique peptides identified.

D. Signal (area as measured by mass-spectrometry) correlation between proteins having an enrichment score $>1$ in the two skeletal muscle samples. Dot size indicates minimal number 
of unique peptides identified in either of the experiments. Proteins detected in only one sample were set to zero at the other. Large dot is Titin.

E. A Venn diagram showing the number of skeletal muscle proteins identified by at least 2 peptides having an enrichment score $>1$, and how many of these proteins were identified (without any filtering criteria) in smooth muscle and adipose tissue. Diagram not to scale. F. Immunofluorescence showing sarcoglycan (SGCA, green) localized to the nuclear periphery (DNA stained with DAPI in blue) in mouse skeletal muscle.

Scale bar - $10 \mu \mathrm{m}$. 
A

B

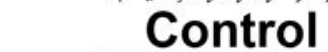

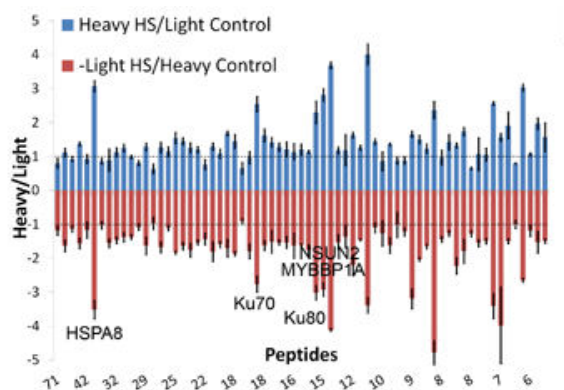

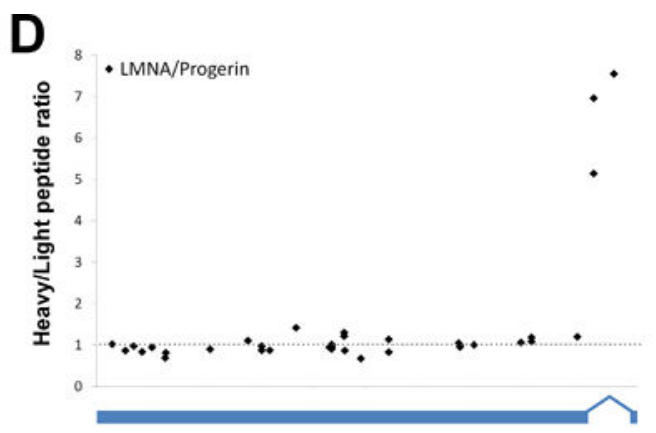

C

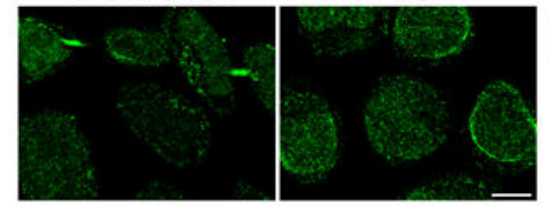

Ku70

Lamin A/C DNA

\section{LMNA}

HeLa

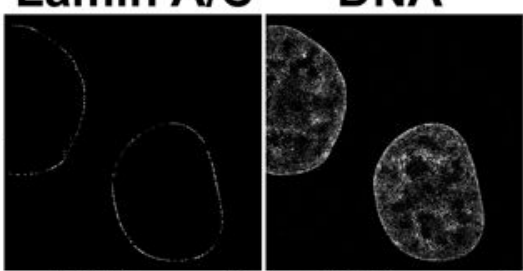

$\mathbf{F}$

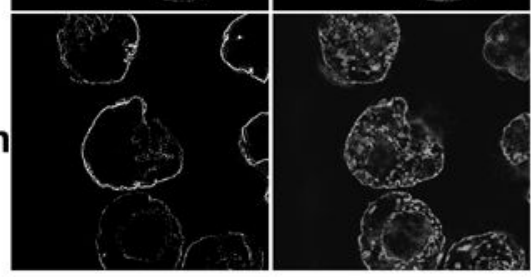

Progerin

\section{Control}

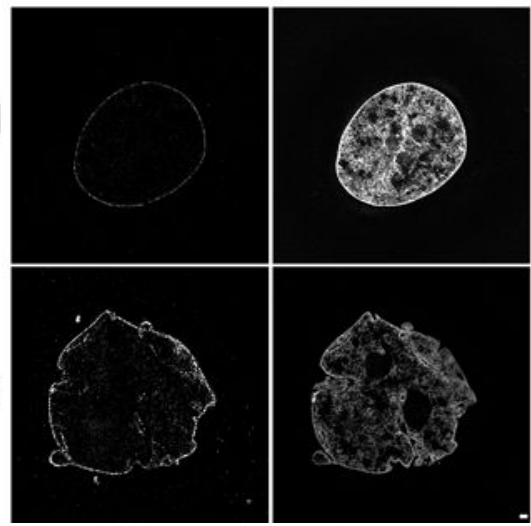

Fib.

HGPS

Figure 3. Differential proteomics

E

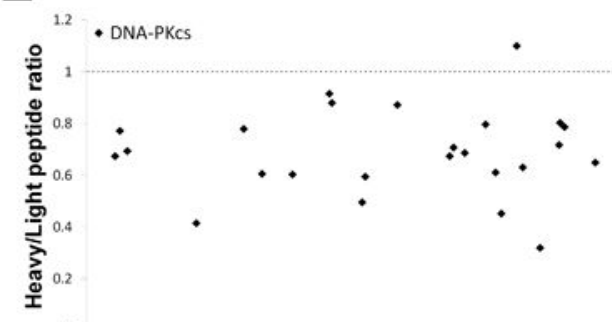

G

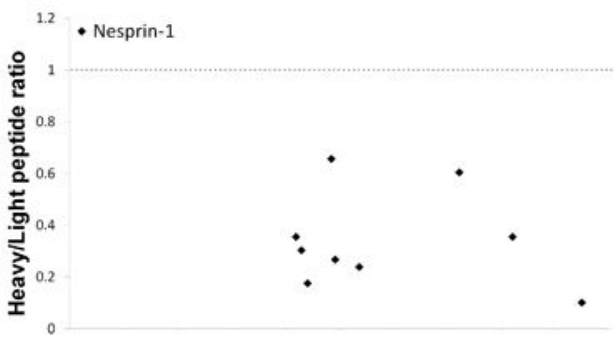

H

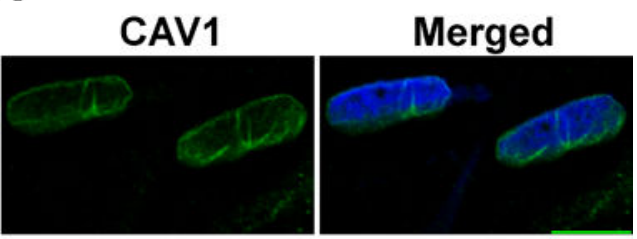

A. Heavy/light ratio of HeLa NE proteins in untreated versus heat shocked cells. Proteins are ordered by sum of peptides used to calculate the heavy/light ratio. The error bars represent heavy/light variability, as calculated by Proteome Discoverer (see methods). $\mathrm{p}=10^{-5} ; \mathrm{n}=10$ and $\mathrm{p}=0.0005 ; \mathrm{n}=8$ for Ku70. $\mathrm{p}=0.007 ; \mathrm{n}=7$ and $\mathrm{p}=0.002 ; \mathrm{n}=8$ for Ku80.

B. Ku70 subcellular localization in HeLa cells, visualized with immunofluorescence, before and after heat shock. Scale bar - $10 \mu \mathrm{m}$. 
C. Structured illumination super-resolution microscopy of HeLa cells transfected with GFPLMNA or GFP-Progerin. Nuclear envelope visualized with GFP.

D. Heavy (lamin A/C) to light (progerin) peptide ratio of GFP-LMNA/Progerin in transfected HeLa cells. X-axis indicates peptide location along the protein. Progerin protein model (blue) marks the location of the 50aa deletion, overlapping with the last 3 peptides. E. Heavy (lamin A/C) to light (progerin) DNA-PKcs peptide ratio in GFP-LMNA/Progerin transfected HeLa cells. X-axis indicates peptide location along the protein.

F. Structured illumination super-resolution microscopy of control and HGPS-derived fibroblasts. Nuclear envelope visualized with a lamin A/C antibody. Scale bar - $1 \mu \mathrm{m}$. G. Nesprin-1 peptide ratio in control (heavy) vs HGPS (light) fibroblasts. X-axis indicates peptide location along the protein.

H. CAV1 immunofluorescence in primary human muscle tissue. Scale bar - $10 \mu \mathrm{m}$. 